Available online at GSC Online Press Directory

GSC Biological and Pharmaceutical Sciences

e-ISSN: 2581-3250, CODEN (USA): GBPSC2

Journal homepage: https://www.gsconlinepress.com/journals/gscbps

(RESEARCH ARTICLE)

\title{
Incidence of blood transfusion reactions in Eritrea: a retrospective cross-sectional study
}

Senet Awolker 1, Yohannes Tekeste 1, Daniel Tesfamichael 1, Selamawit Nemariam ${ }^{1}$, Nahom Yacob 1, Yohana Mehreteab ${ }^{1}$, Selomon Eyob ${ }^{1}$ and Mulugeta Russom 2,*

${ }^{1}$ National Blood Transfusion Center, Ministry of Health, Asmara, Eritrea.
${ }^{2}$ Eritrean Pharmacovigilance Centre, National Medicines and Food Administration, Ministry of Health, Asmara, Eritrea.

Publication history: Received on 07 August 2020; revised on 21 August 2020; accepted on 26 August 2020

Article DOI: https://doi.org/10.30574/gscbps.2020.12.2.0257

\begin{abstract}
Blood Transfusion is among the life-saving medical interventions that has significant impact in preventing and/or reducing mortality. It has however been associated with life-threatening adverse outcomes. In Eritrea, the burden of blood transfusion reaction is unknown. This study is conducted to estimate the incidence and determine the nature of blood transfusion reactions in all Eritrean hospitals using historical data. This was a retrospective descriptive crosssectional study conducted in all Eritrean hospitals. Data was collected from all Eritrean hospitals', Eritrean Pharmacovigilance Centre and the National Blood Transfusion Centre in Eritrea and entered and analyzed using CSPro and SPSS respectively. In the ten years period, a total of 87,655 blood and blood components were transfused and 37 cases of blood transfusion reactions ( 0.42 cases per 1000 transfusions) were documented. The median age of the study population was 27 years (interquartile range: 23 ) and reactions were mainly reported in females (68\%). All but one of the reported blood transfusion reactions were acute and dominated by immunogenic febrile non-hemolytic transfusion reaction (37.8\%) followed by allergic reaction (29.7\%), anaphylaxis (10.8\%), and hemolytic transfusion reaction other than $\mathrm{ABO}$ antibodies (8.1\%). Almost all reactions were acute and majority of which were febrile non-hemolytic transfusion reaction. About one in four (24.3\%) of the cases were serious and outcome was fatal in two cases. In conclusion, though the estimated incidence of blood transfusion reaction in Eritrea was found to be low, the seriousness of the cases urge establishment of a functional haemovigilance program.
\end{abstract}

Keywords: Blood transfusion; Adverse reactions; Incidence; Haemovigilance

\section{Introduction}

Blood transfusion, a life-saving medical intervention, has been associated with mild to serious adverse reactions. The occurrence of acute blood transfusion reactions has been estimated to be between $0.2 \%$ and $10 \%$ with fatal outcome of approximately one in 250,000 transfusions [1]. Aimed at detecting, reporting, managing and preventing blood transfusion related reactions, efforts has been made by different countries to establish functional haemovigilance systems. Haemovigilance is defined as "a set of surveillance procedures covering the whole transfusion chain (from the collection of blood and its components to the follow-up of recipients), intended to collect and assess information on unexpected or undesirable effects resulting from the therapeutic use of labile blood products, and to prevent their occurrence or recurrence" [2].

In Eritrea, a National Blood Transfusion Center (NBTC) was established in January 2002 [3]. The Centre collects blood from voluntary non-remunerated blood donors and family replacement donors' around 8,000 - 10,000 units annually. To ensure blood transfusion safety, all the collected blood units are tested for ABO and Rh blood grouping, screens for

\footnotetext{
* Corresponding author: Mulugeta Russom, e-mail: satiswt@gmail.com

Eritrean Pharmacovigilance Centre, National Medicines and Food Administration, Ministry of Health, Asmara, Eritrea.
} 
infectious markers (HIV, HBsAg, HCV and Syphilis), prepares for different blood components and finally distributes blood and blood components to hospitals as required. With the implementation of quality systems in 2008, all hospitals practicing in blood and blood component transfusions in Eritrea were informed to detect and report blood transfusion reactions (BTRs) and reporting forms were distributed to all hospitals. Despite this, limited cases of BTRs have been reported to the NBTC. The Centre's assumption was that, there are BTRs but might be left under-reported. This study was therefore conducted to retrospectively assess the incidence and nature of blood transfusion reactions in all hospitals in Eritrea in the last ten years (2008 - 2017).

\section{Methods}

\subsection{Study design and setting}

A retrospective cross-sectional study was carried out to measure the incidence of blood transfusion reactions (BTRs) in 25 hospitals in Eritrea documented between January 2008 and December 2017. Data from all Eritrean hospitals was collected by the research team from September to November 2018.

\subsection{Study population}

All patients transfused with blood and blood components in all Eritrean hospitals between January 2008 and December 2017 were considered as study populations for this study.

\subsection{Data sources}

For this study, patients' clinical records, laboratory registries, databases of the Eritrean Pharmacovigilance Centre and National Blood Transfusion Centre reports were used as data sources.

\subsection{Exposure and outcome definition}

The exposure of this study was transfused blood components. Blood is collected from healthy individuals and is stored in blood banks for transfusion in life-threatening conditions. The collected whole blood units were separated into components and transfused as per the recipients' requirement. The blood components used were red blood cells, platelets and fresh frozen plasmas.

The primary outcome of this study was documented adverse reactions related to blood transfusion including their outcomes. Seriousness of the event was determined using the ICH definition [4]. According to the ICH definition, a reaction was considered as serious if it results in death, or is life-threatening, requires hospitalization/prolongation of hospitalization, results in persistent or permanent disability, congenital abnormality or other medically important disease condition that if not intervened could lead to one of the above-mentioned conditions.

\subsection{Data collection approach}

A questionnaire adopted from the existing blood transfusion reaction reporting form was used to collect data [S1 data collection tool]. Data on BTR was collected from all laboratory registries of respective hospitals. Besides, BTRs reported to the Eritrean Pharmacovigilance Centre and National Blood Transfusion Centre during the ten years period were collected. To avoid duplicate cases, patient initials, health facility, date reaction started and stopped and date of blood transfused were considered.

\subsection{Statistical analysis}

Data was analyzed using Statistical Package for Social Sciences (SPSS version 20). The description of frequencies, proportions, and cross tabulations of the data for each variable was calculated for the independent variables like age, sex, and region and the dependent variables of different types of blood transfusion reactions. Incidence of BTR rate was calculated per 1000 patients transfused. The cases were classified based on the clinical features reported by attending physician as well as the laboratory findings.

\subsection{Ethical considerations}

Ethical clearance was obtained from the health research protocol review and ethical clearance committee of the Ministry of Health. Besides, official letter was sent to the Zonal Medical Officers and Medical Directors of all respective zones and hospitals respectively to allow us to conduct the study. Due to the retrospective nature of the study, patient 
consent was waived and professional ethics was followed by the researchers to keep patient information strictly confidential.

\section{Results}

From 2008 to 2017, a total of 87,655 units of blood and blood components were transfused to patients in various health facilities for different cases. Transfused units were dominated by RBC 43,143 followed by whole blood 37,597 (Figure 1). Of the 25 hospitals, blood transfusion reaction reporting forms were available in 12 (48\%) hospitals and only three hospitals were found to use it.

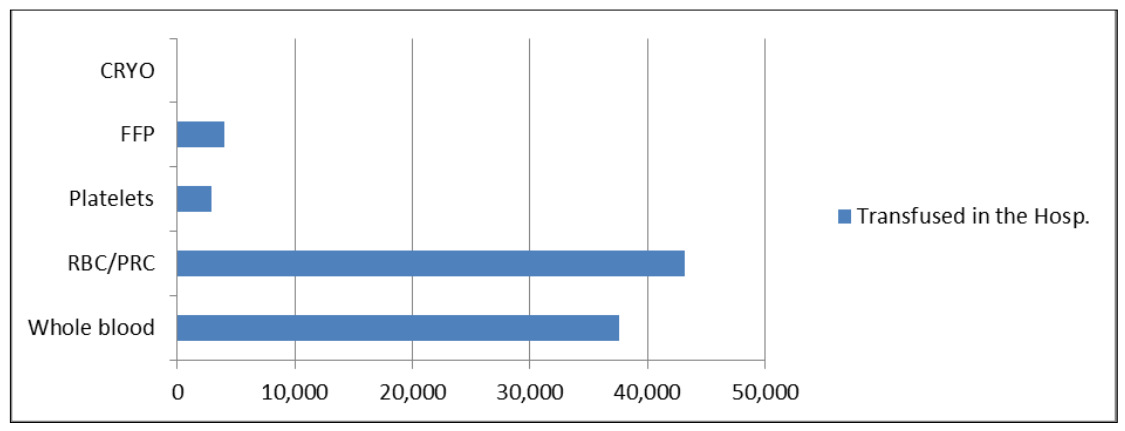

Figure 1 Blood components transfused between 2008 and 2017, Eritrea

In the ten-year period, 37 cases of blood transfusion reactions were reported from nine hospitals. The cases were dominated by females (68\%) and their median age was found to be 27 years (IQR: 23). Blood group was reported in 27 of the cases and majority of them were found to be $0^{+}(73 \%)$ and $\mathrm{A}^{+}(22.2 \%)$.

Table 1 Details of blood transfusion reactions captured between 2008 and 2017Eritrea

\begin{tabular}{|c|c|c|c|c|c|}
\hline Types of reaction & Reaction details & Male & Female & Total & Percent (\%) \\
\hline \multirow{10}{*}{$\begin{array}{l}\text { Acute transfusion } \\
\text { reactions }\end{array}$} & Immunologic & & & & \\
\hline & FNHTR & 6 & 8 & 14 & 37.8 \\
\hline & Allergic reaction & 3 & 8 & 11 & 29.7 \\
\hline & Anaphylaxis/hypersensitivity & 0 & 4 & 4 & 10.8 \\
\hline & HTR (due to irregular ant-bodies) & 1 & 2 & 3 & 8.1 \\
\hline & Non-Immunologic & & & & \\
\hline & Hypotension & 0 & 2 & 2 & 5.4 \\
\hline & TACO & 1 & 0 & 1 & 2.7 \\
\hline & Respiratory distress & 1 & 0 & 1 & 2.7 \\
\hline & Unspecified notification & - & 1 & 1 & 2.7 \\
\hline \multirow{9}{*}{$\begin{array}{l}\text { Details of the } \\
\text { blood transfusion } \\
\text { reactions } \begin{array}{l}\text { with } \\
\text { signs and } \\
\text { symptoms }\end{array}\end{array}$} & Pyrexia & 7 & 10 & 17 & 24.6 \\
\hline & Rash/allergic reaction & 6 & 10 & 16 & 23.2 \\
\hline & Tachycardia & 5 & 10 & 15 & 21.7 \\
\hline & Rigor & 6 & 3 & 9 & 13.0 \\
\hline & Vomiting & 1 & 3 & 4 & 5.8 \\
\hline & Haemoglobinuria & 1 & 2 & 3 & 4.3 \\
\hline & Positive DAT & 0 & 1 & 1 & 1.4 \\
\hline & Lumbar pain & 1 & 2 & 3 & 4.3 \\
\hline & Oliguria/Anuria & 0 & 1 & 1 & 1.4 \\
\hline \multirow{3}{*}{$\begin{array}{l}\text { Type of blood } \\
\text { product identified }\end{array}$} & Whole blood & 6 & 20 & 26 & 70.3 \\
\hline & $\mathrm{RBC} / \mathrm{PRC}$ & 6 & 5 & 11 & 29.7 \\
\hline & Total & 12 & 25 & 37 & 100.0 \\
\hline
\end{tabular}

FNHTR: Febrile non-hemolytic transfusion reaction; HTR: Hemolytic transfusion reaction; TACO: Transfusion associated circulatory overload; DAT: Direct anti-globulin test; RBC: Red blood cells; PRC: Packed red blood cells. 
All but one of the reported blood transfusion reactions were acute and dominated by immunogenic febrile nonhemolytic transfusion reaction (37.8\%) followed by allergic reaction (29.7\%), anaphylaxis (10.8\%), and hemolytic transfusion reaction other than ABO antibodies (8.1\%) (Table 1). The non-immunogenic reactions were dominated by hypotension (5.4\%) followed by transfusion associated circulatory overload $(2.7 \%)$ and respiratory distress $(2.7 \%)$. One of the reports was difficult to specify its type due to the limited information in the report form. Of the 37 cases of BTRs, 26 were after whole blood transfusion and the remaining 11 were encountered following RBC transfusion.

In this study, the estimated incidence rate of blood transfusion reaction per 1000 transfused units of whole blood and $\mathrm{RBC} / \mathrm{PRC}$ was 0.69 and 0.25 respectively. There was no reaction reported related to platelets and fresh frozen plasma transfusions. This gives an overall estimated incidence of 0.42 cases per 1000 transfused blood and blood components in the last ten years (Table 2).

Table 2 Estimated incidence rate of blood transfusion reactions (BTRs) per 1000 transfused blood and blood components, 2008 - 2017, Eritrea.

\begin{tabular}{|l|l|l|l|}
\hline $\begin{array}{l}\text { Blood } \\
\text { Component }\end{array}$ & $\begin{array}{l}\text { Transfused blood and } \\
\text { blood components }\end{array}$ & Number of BTRs & $\begin{array}{l}\text { Estimated incidence rate per } \\
\mathbf{1 0 0 0} \text { transfusions }\end{array}$ \\
\hline Whole Blood & 37,597 & 26 & 0.69 \\
\hline RBC/PRC & 43,143 & 11 & 0.25 \\
\hline Platelets & 2,942 & 0 & 0.00 \\
\hline FFP & 3,973 & 0 & 0.00 \\
\hline Overall & 87,655 & 37 & 0.42 \\
\hline
\end{tabular}

Majority of the cases were reported in $2012(\mathrm{n}=11)$ followed by $2017(\mathrm{n}=10)$ and $2016(\mathrm{n}=8)$. All cases of anaphylaxis $(\mathrm{n}=4)$ were reported solely in 2017 . Overall, majority of the cases $(83.8 \%)$ were found to be non-serious. The rest, $16.2 \%$, were serious.

\section{Discussion}

In this study, the estimated incidence of blood transfusion reaction in Eritrea was found to be low. This finding is consistent with studies reported from Zimbabwe (0.46/1000) [2], Indian Institute of Medical Sciences (0.5/1000) [5] and South Africa (0.49/1000) [6] but much lower than findings from Switzerland (4.2/1000) [7], France (3/1000) [8] and another Indian study (9.2/1000) [9]. From this, it can be assumed that countries with functional haemovigilance system detect much higher rates of blood transfusion reactions compared to the developing countries for obvious reasons. In settings where matured haemovigilance system is not in place, many of the incidents are left under-reported that ultimately lead to under-estimation of blood transfusion related risks. This might be the main reason, among others, for the low blood transfusion reaction reports in Eritrea as only three hospitals were using the reporting forms. Otherwise, with the immature and overstretched healthcare system in low-income countries, one would expect higher incidents compared to that of developed ones. A similar but an observational study conducted in Nigeria however reported entirely incomparable results (87 cases per 1000) [10]; possibly due to its prospective nature. This urges researchers to conduct prospective studies to better estimate the incidence of blood transfusion reactions as majority of the aforementioned findings are from retrospective studies.

Almost all blood transfusion reactions reported in this study were acute and majority of which were febrile nonhemolytic transfusion reaction (FNHTR) followed by allergic reactions. This is more or less similar with the findings from Zimbabwe [2] and Nigeria [10] in which 58.5\% and 65\% of the cases were found to be FNHTR respectively. Whereas, two of the Indian studies mentioned-above found allergic reactions as the most frequently reported incidents followed by FNHTR [5,9]. Another event of interest of blood transfusion reaction is anaphylaxis; which is lifethreatening in nature. Thus, healthcare professionals should be ultra-cautious on the risk of anaphylactic reactions and should be well prepared to monitor and manage such serious adverse events at the earliest. 
In this study, two of the documented cases were found to be fatal. The first case was a 15-month old, male child, admitted with a diagnosis of malnutrition and gastroenteritis. On his $8^{\text {th }}$ day of admission, the child transfused with $100 \mathrm{ml}$ of whole blood for the management of severe anemia (hemoglobin level 4.8). Three hours following administration of the whole blood, the patient reached critical with circulatory over load and died. The second death case was a 55 years old male patient admitted with the diagnosis of plasmodium falciparum malaria and severe anemia and was taking quinine sulphate intravenously. Patient transfused with two units of whole blood and developed respiratory distress and died at the same day.

Ideally, serious blood transfusion events including deaths should be investigated and assessed at the earliest to confirm the diagnosis and determine causality. As there was no functional haemovigilance system in the country, the cases were left uninvestigated. Hence, contribution of their underling illness and/or other possible factors could not be fully ruled out. Establishment of haemovigilance program in Eritrea is therefore vital to ensure blood transfusion safety and avoid blood transfusion reaction related deaths which could compromise public confidence on the healthcare system. As part of the quality management systems, introduction of mechanisms of detection, reporting, investigation of blood transfusion reactions and taking corrective and preventive actions at the earliest possible time has paramount importance.

Female patients taking whole blood and those with $\mathrm{Rh}$ positive blood group types (mainly $\mathrm{O}^{+}$) were more frequent to develop blood transfusion reactions in this study. This can be explained by the fact that these group of people were highly exposed to blood transfusion and thus, more likely to experience blood transfusion reactions. Besides, the high predominance of blood transfusion reactions in females could be explained with antibodies developed during pregnancy and/or previous transfusions.

The main inherent limitation of this study was its retrospective nature. In countries like Eritrea where electronic medical records and haemovigilance system are not in place, much of the cases could be left under-reported and thus, the problem might be under-estimated. Besides, detailed demographic characteristics of transfused patients were not adequately documented in the laboratory registries which limited us to identify possible risk factors that predispose patients to blood transfusion reactions.

\section{Conclusion}

The estimated incidence of blood transfusion reaction in Eritrea was found to be low, but the few fatal and lifethreatening adverse reactions urge the establishment of a functional haemovigilance program in Eritrea. To ensure blood transfusion safety, continued training of healthcare professionals on how to detect, report, manage, investigate and assess blood transfusion related problems and close monitoring/supervision are important.

\section{Compliance with ethical standards}

\section{Acknowledgments}

The authors sincerely acknowledge the National Medicines and Food Administration of the Ministry of Health for their financial support to conduct the study. The continued collaboration of Zonal Medical officers, Zonal Pharmacy Services, Laboratory Technicians and Medical Directors of the respective institutions is much appreciated. We also thank Andebrhan Tewolde, Statistician, for his valuable assistance during the data analysis stage.

\section{Disclosure of conflict of interest}

The authors declare that they have no competing interests.

\section{Statement of ethical approval}

The present research work does not contain any studies performed on animals/humans subjects by any of the authors.

\section{Statement of informed consent}

Not applicable. 


\section{References}

[1] Khalid S, Usman M and Khurshid M. (2010). Acute transfusion reactions encountered in patients at a tertiary care center. J Pak Med Assoc, 60(10), 832-836.

[2] Mafirakureva N, Khoza S, Mvere D, et al. (2014). Incidence and pattern of 12 years of reported transfusion adverse events in Zimbabwe: a retrospective analysis. Blood Transfus, 12(3), 362-367.

[3] Ministry of Heath of Eritrea. National Blood Transfusion Services. Quality System Manual and Site Master File. (2002). Asmara, Eritrea.

[4] International Conference for Harmonization. (2003). Post-approval safety data management: definitions and standards for expedited reporting (E2D). ICH harmonized tripartite Guidelines, version 4.

[5] Kumar P, Thapliyal R, Coshic P and Chatterjee K. (2013). Retrospective evaluation of adverse transfusion reactions following blood product transfusion from a tertiary care hospital: A preliminary step towards haemovigilance India. Asian J Transfuse Sci, 7(2), 109-15.

[6] South Africa National Blood Services. (2007). Haemovigilance Report 2007. South Africa National Blood Service, South Africa.

[7] Michlig C, Vu DH, Wasserfallen JB, et al. (2003). Three years of haemovigilance in a general university hospital. Transfus Med, 13, 63-72.

[8] Andreu G, Morel P, Forestier F, et al. (2002). Hemovigilance network in France: organization and analysis of immediate transfusion incident reports from 1994 to 1998. Transfusion, 42, 1356-64.

[9] Sharma DK, Datta S and Gupta A. (2015). Study of acute transfusion reactions in a teaching hospital of Sikkim: A hemovigilance initiative; Indian J Pharmacol, 47(4), 370-374.

[10] Arewa OP, Akinola NO and Salawu L. (2009). Blood transfusion reactions: evaluation of 462 transfusions at a tertiary hospital in Nigeria. Afr J Med Sci, 38(2), 143-8. 